local orientation of surfaces and their reflectance. Again, in deciding how to represent the 3-D model of an object, Marr is guided by a computational theory of the process of recognition. First, the representation has to be accessible, that is, it must be possible to compute it economically from the image. Second, it should have a wide scope in that it must be possible to represent any object that can be recognized. Third, it must provide a unique description of a given object, otherwise different descriptions would be formed on different occasions and hence recognition would fail. Fourth, the representation should reflect the similarity between similar objects, but should also make explicit small differences: we can both see the similarity between different people and can also recognize individuals. Marr's method of representing 3-D objects meets all these constraints. Thus, the last constraint is satisfied by organizing the representation hierarchically so that the coarse shape of an object is represented in one module with other modules representing successively finer and finer detail (see diagram p.693).

There is a further way in which Marr's work is superior to that of most of his contemporaries. Other vision programs have usually taken no account of psychological or neurophysiological findings. In deciding what algorithm to employ, Marr makes use of both kinds of

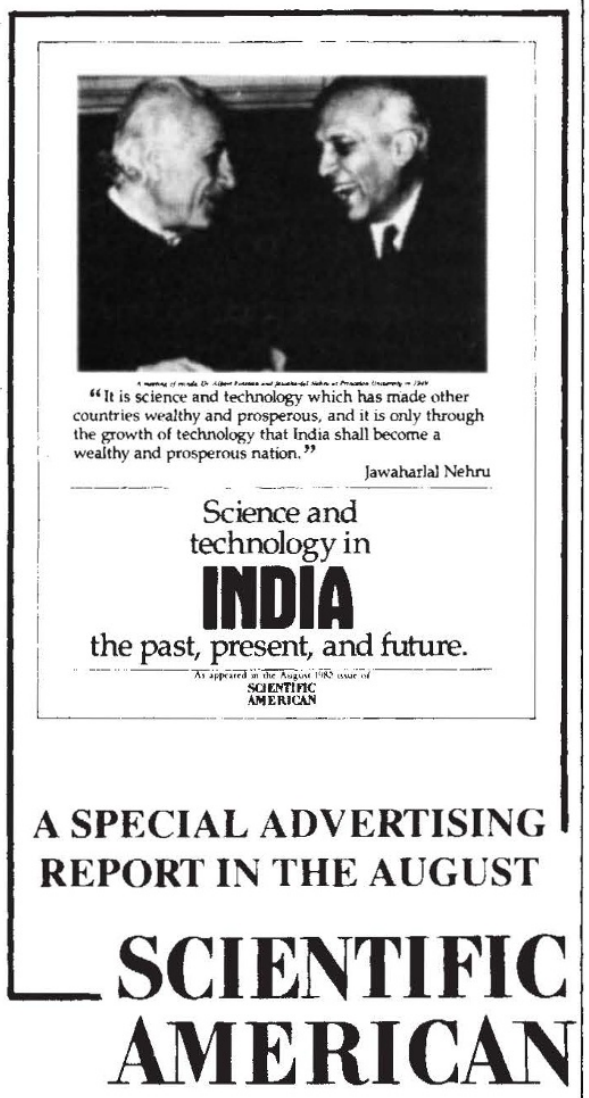

Circle No. 10 on Reader Service Card evidence where relevant results exist. For example, having proposed a simple and elegant model of stereopsis, he abandoned it because it did not fit the psychophysical findings. He put forward a second model, which although considerably more complex is consistent with the psychophysical results. In essence it begins by matching large features in the two images and allows for the use of eye-movements to reduce disparities in matching finer detail. Again, in his account of motion perception, he suggests that Y-cells may subtract a measure of the brightness at time $t+60 \mathrm{msec}$ from the same measure at time $t$ : hence a cell will fire only if an edge is moving with the lighter part leading. $\mathrm{He}$ makes out a case that this idea is consistent with the known physiological properties of $\mathrm{Y}$-cells. This is an ingenious idea for motion detection, but there is so much confusion amongst neurophysiologists about the role of X-and Y-cells that it may be premature.

Vision has two faults. First, despite Marr's emphasis on the importance of implementing algorithms as computer programs to make sure they work, it is often unclear whether he has actually written and tested a computer program to perform a particular task. Moreover, even when he claims to have implemented an algorithm, he rarely discusses how successful the program is and he gives no clear indication of the range of scenes on which it succeeds and those on which it fails. This nonchalant approach to computer modelling is unfortunately typical of the Artificial Intelligence community. Second, he is sometimes unduly dismissive of the research of others. For example, he scorns the work of Waltz and Mackworth, who wrote programs to recover depth from polyhedra, on the grounds that they were dealing with "miniworlds". His allegation is correct, but it remains true that people can readily see depth in line-drawings of such objects and Marr provides no alternative account of how this task is performed. Both Waltz and Mackworth in fact succeeded (at different levels) in working out the constraints that enable this task to be carried out and to that extent they were amongst the few of Marr's predecessors who had a computational theory of the task they simulated.

Marr begins his preface by writing "This book is meant to be enjoyed": his intention will be fulfilled. The book is not only enjoyable, it is exciting and contains many stimulating ideas. For example, he gives good reasons for supposing that the brain cannot use iterative processes unless they converge very fast. Vision is perhaps the most important book on the subject to appear since Helmholtz's Physiological Optics was published over a hundred years ago. Marr's untimely death was a grievous loss to psychology.

Stuart Sutherland is Director of the Centre for Research on Perception and Cognition at the University of Sussex.
NMR of biopolymers

\author{
Alfred G. Redfield
}

NMR in Molecular Biology. By Oleg Jardetzky and G. C. K. Roberts. Pp.681. ISBN 0-12-380580-5. (Academic: 1982.) $\$ 59, £ 39$.

AlTHOUGH nuclear magnetic resonance does not occupy the central role in the biological sciences that it has in analytical organic chemistry, there is hardly a branch of biochemistry or biology to which it has not been applied in recent years. The physical basis for these biological applications is the same as for organic chemistry, but the goals and general methodology differ significantly. While no unusually subtle new ideas are used, biologically relevant NMR is often complicated and controversial in its interpretation, owing to the complexity of the systems involved, and it forms a new and flourishing sub-discipline. Most current research is directed toward finegrained local structural studies, and the determination of dynamic behaviour which only NMR can provide.

The authors of this treatise were among the innovators, and continue to be active, in this field. Their book is a thorough treatment of the most mature application of NMR to biological problems, namely the study of small molecules and macromolecules in solution, alone and in interaction with each other. Topics such as in vivo metabolism and NMR imaging are omitted, as is a comprehensive treatment of NMR of partly immobilized or frozen structures (commonly called "solid state NMR") except for a brief description of its application to membranes and related problems. The emphasis is therefore on proteins, especially enzymes, reflecting both the majority of current applications and the experience of the authors. Restricting the content of the book in this way was wise, since the areas not covered were in early stages of development at the time (early 1981) when the book was completed, and because the treatment of solution-state NMR could then be comprehensive in a book of reasonable length.

There are short introductory sections on fundamentals and instrumentation, and also descriptions of theories of relaxation, both paramagnetic and diamagnetic. Most

\section{Climate and history}

Nineteen eighty two seems to be the year for books on climate and history. In addition to the two volumes reviewed by Stephen Schneider (Nature 298, 499) are Climatic Change in Later Prehistory, edited by Anthony Harding and published by Edinburgh University Press, and FoodClimate Interactions with Wilfrid Bach et al. as editors and Reidel as publisher. Both books are the records of meetings. Prices are (EUP) pbk £9.75, (Reidel) hbk Dfl. 135, \$58; pbk Dfl. $65, \$ 28.50$. 